\title{
Bariatric Surgery and Its Effects on Calcium and Bone Metabolism
}

\author{
Susan E. Williams
}

Published online: 17 October 2014

(c) Springer Science+Business Media New York 2014

The prevalence of obesity in the United States rapidly reached pandemic proportions during the second half of the twentieth century. Paralleling the pandemic, surgical treatment approaches came into vogue, stemming at least in part from the fact that patients who had undergone intestinal resection experienced significant and durable weight loss. Recent data from the American Society for Metabolic \& Bariatric Surgery noted that the number of bariatric surgeries performed in the U.S. rose from 13,365 in 1998 to an estimated 220,000 in 2009, and has continued to rise.

Documented nutritional 'disturbances' and weight loss following resection of major portions of the small intestine began to emerge very early in the twentieth century. J. D. Whitall in his 1911 paper examined outcomes involving various lengths of intestinal resection and concluded that the patient's prognosis hinged upon several key factors, not the least of which was the location of the portion removed. Two decades later publications started to emerge that documented profound cases of hypocalcemia, tetany, and osteomalacia following massive resections of small intestine.

It is pure speculation as to if an "Aha moment" occurred leading up to the first surgeries exclusively to treat obesity. However, in the United States during the 1950s, bypass surgeries known as 'intestinal short circuiting' were being investigated. The earliest bariatric procedures bypassed a great deal more of the small intestine than modern day procedures and did indeed result in dramatic weight loss

\section{S. E. Williams $(\square)$}

Department of Endocrinology, Diabetes and Metabolism,

Cleveland Clinic, 10685 Carnegie Avenue/X-20, Cleveland, OH 44106, USA

e-mail: WilliaS9@ccf.org but were accompanied by severe diarrhea, electrolyte imbalance, hepatic failure, and a high rate of mortality.

Since that time myriad advances in the field of bariatric surgery have addressed many of the more serious postoperative metabolic complications and today there are several safe, effective bariatric procedures. However, the untoward consequences of malabsorption and dramatic weight loss on skeletal health persist to the present day.

In this edition of CRBMM our authors present current knowledge in this unique field and discuss what is known as well as what remains unknown in the understanding, diagnosis, prevention, and treatment of metabolic bone disease in this population.

An historical overview sets the stage for this edition, highlighting fundamental discoveries that began to link extensive gut loss to the dire consequences of metabolic bone disease. This article is followed by an excellent discussion by Jacqueline Center's group exploring not only the limited evidence regarding the mechanisms of increased bone turnover but also some of the technical challenges of measuring bone during periods of severe weight loss.

Peters et al. define known risk factors for secondary hyperparathyroidism in this population and discussed the benefits as well as the limitations of preventive strategies that include adequate dietary protein.

Pramyothin and Holick explain the relationship between obesity and vitamin D deficiency, how weight loss affects 25-hydroxyvitamin D levels, and why despite massive weight loss, aggressive supplementation remains the mainstay of treatment.

Finally, Sakhaee provides a detailed review of current knowledge of post bariatric surgery bone changes including mechanisms of bone loss and the role of calcitropic hormones. The high prevalence of hyperoxaluria, renal stone 
formation, and the proposed underlying mechanisms are also discussed in detail. She provides an insightful critical review of the current practice guidelines and challenges the standard practice of calcium citrate supplementation. And in closing she highlights the paucity of hard evidence defining an efficacious approach to the prevention and treatment of metabolic bone disease in this most challenging patient population.

As I reviewed each of these articles I was struck by the incongruent evidence as well as the want for unified, efficacious clinical guidelines. Despite numerous scientific investigations, optimal management of this patient population-both before and after bariatric surgeryremains elusive. The authors captured well the current evidence and the need for further investigation while revealing much about the complex pathophysiologic mechanisms that are involved in vitamin D deficiency, bariatric osteomalacia, hyperoxaluria, and nephrolithiasis.

Undoubtedly there will come a day when surgical intervention for morbid obesity will be a thing of the past but for now, for the thousands of patients affected, we must continue to seek to define efficacious preventive strategies and treatment protocols for metabolic bone disease in bariatric surgery patients. 